\title{
Nanostructure of organic semiconductor thin films: Molecular dynamics modeling with solvent evaporation
}

\author{
Gertsen, Anders Skovbo; Sørensen, Michael Korning; Andreasen, Jens Wenzel
}

Published in:

Physical Review Materials

Link to article, DOI:

10.1103/PhysRevMaterials.4.075405

Publication date:

2020

Document Version

Publisher's PDF, also known as Version of record

Link back to DTU Orbit

Citation (APA):

Gertsen, A. S., Sørensen, M. K., \& Andreasen, J. W. (2020). Nanostructure of organic semiconductor thin films: Molecular dynamics modeling with solvent evaporation. Physical Review Materials, 4(7), [075405].

https://doi.org/10.1103/PhysRevMaterials.4.075405

\section{General rights}

Copyright and moral rights for the publications made accessible in the public portal are retained by the authors and/or other copyright owners and it is a condition of accessing publications that users recognise and abide by the legal requirements associated with these rights.

- Users may download and print one copy of any publication from the public portal for the purpose of private study or research.

- You may not further distribute the material or use it for any profit-making activity or commercial gain

- You may freely distribute the URL identifying the publication in the public portal 


\title{
Nanostructure of organic semiconductor thin films: Molecular dynamics modeling with solvent evaporation
}

\author{
Anders S. Gertsen $\odot$, Michael Korning Sørensen, and Jens W. Andreasen ${ }^{*}{ }^{*}$ \\ Department of Energy Conversion and Storage, Technical University of Denmark, Fysikvej 310, DK-2800 Kgs. Lyngby, Denmark
}

(Received 12 March 2020; accepted 7 July 2020; published 29 July 2020)

\begin{abstract}
We present a procedure for simulating solution deposition of organic thin-films on explicitly modeled substrates via solvent evaporation simulations in a molecular dynamics framework. Additionally, we have developed force fields for the family of IDTBR nonfullerene acceptors, which have been widely employed in the literature as $n$-type materials in several types of organic semiconductor devices, and we analyzed their structure-property relationships using a combination of grazing incidence $\mathrm{x}$-ray scattering measurements, atomistic molecular dynamics simulations, and quantum chemical calculations. We find that thermal fluctuations can have a significant impact on calculated electron transfer integrals, and that the $\pi$-stacking interactions of the electron withdrawing benzothiadiazole building blocks are key to high electron coupling in amorphous thin films of $n$-type materials.
\end{abstract}

DOI: 10.1103/PhysRevMaterials.4.075405

\section{INTRODUCTION}

Organic photovoltaics (OPVs) represent a promising technology for low-cost, lightweight, flexible solar cells with remarkably low energy payback times when deposited using scalable, roll-to-roll compatible fabrication techniques [1-3]. Traditionally, OPVs have utilized fullerene derivatives as electron acceptors, but the emergence of nonfullerene, smallmolecule acceptors (NFAs) and the advances that these have carried with them in recent years are testament to their present and, not least, future importance in the field [4,5]. Record efficiencies of more than $18 \%$ have been reached when utilizing NFAs [6], surpassing those of fullerene-based devices due to, e.g., lower voltage losses and higher current generation [7]. Several accompanying and equally important advantages of NFA OPVs include the significant reduction of performance losses within the first few days or weeks under illumination (i.e., reduced burn-in) $[8,9]$ as well as impressive thermal stabilities [10-12]. These latter two properties are influenced not only by the differences in electronic properties of NFAs and fullerenes, but also by their morphological differences [10,13-16].

Experimental morphology studies on organic thin-films can be conducted in various ways. The most commonly employed methods are grazing incidence x-ray scattering experiments in wide-angle or small-angle geometries (GIWAXS and GISAXS, respectively). These methods can provide quantitative, statistical information on structural parameters: The former of molecular order such as $\pi$-stacking distances and lamellar stacking distances in both neat films and blend films, and the latter of mesoscale order such as domain sizes in blend films. To interpret data from these measurements, molecular dynamics (MD) simulations can prove helpful by providing

\footnotetext{
*jewa@dtu.dk
}

a more qualitative insight into the short-range structural parameters, and, in combination with quantum chemical calculations, elucidating different aspects of the structure-property relationships $[17,18]$.

In recent years, IDTBR NFAs [see Fig. 1; cf. the supplemental information (SI), Sec. S1 for full names of all compounds mentioned in this paper] [19] have gained significant attention due to the stable, burn-in free, and highly efficient OPV devices based on these [8-10,15,20,21]. Furthermore, it has very recently been shown that the structural packing of IDTBR acceptors deduced from single crystals can explain the high $n$-type mobilities in organic thin-film transistors (OTFTs) of IDTBR derivatives [22]. Common for OPVs and OTFTs is that the active layer film thicknesses are often less than $100 \mathrm{~nm}$, and with air-interface and substrate effects arguably becoming increasingly important with thinner films, it calls for inclusion of these in thin-film simulations [23]. Although very thin-films are favorable in terms of computational cost, the structural relaxation of solution processed thin-films happens on time-scales that are inaccessible to atomistic MD, and a priori knowledge of the molecular packing is thus often necessary [24]. This reduces the predictive power of the simulations. Coarse-grained models of various resolution, i.e., describing a few atoms or up to whole monomers by effective particles or "beads," have successfully been employed to simulate bulk, blend morphologies and their scattering signatures [25-27], but extensive fitting of the coarse-grained force fields to either atomistic simulations or experiments is needed for new materials. Additionally, a too coarse resolution can greatly limit the chemical specificity of the model, in turn hampering further property calculations. In particular, novel donors and NFAs for OPV applications are structurally complex and thus more difficult to coarse-grain than the benchmark system P3HT:PC ${ }_{61} \mathbf{B M}$. The construction of reliable, atomistic models is hence often a prerequisite for reliable coarse-grained models, and with the multitude of interactions 


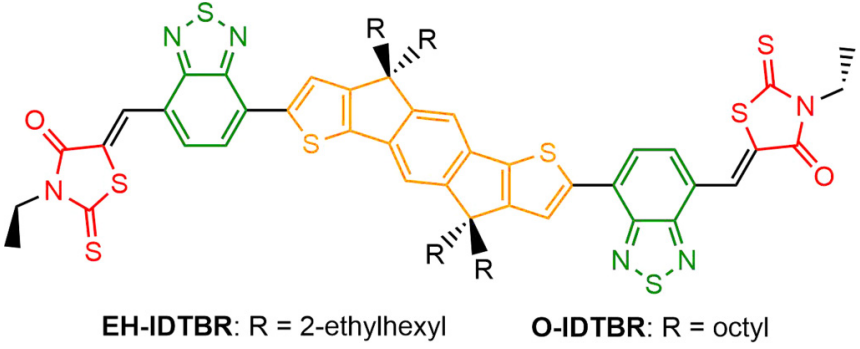

FIG. 1. Structures of EH-IDTBR and O-IDTBR; orange marks the indacenodithiophene (IDT) subunit, green marks the benzothiadiazole (BT), and red marks the rhodanine (RH).

needed for simulating solution processed thin-films due to substrate and air-interface effects, they are a necessary first step.

Different approaches to modeling deposition of organic thin-films in nonequilibrium MD simulations, including vapor deposition and solvent evaporation, have been published during the years [28]. Our work is centered around organic thinfilms processed from solution using deposition techniques such as spin-coating and slot-die coating [3], and the focus herein will thus be on solvent evaporation simulations. Only a few studies on solvent evaporation MD simulations have been published: One of the more complete efforts in trying to capture as many effects as possible simultaneously was published by Peter, Meyer, and Baschnagel [29]. By omitting periodic boundary conditions (PBCs) in the direction of the surface normal and including potential walls at the top and bottom surfaces of the box instead, they tuned the Lennard-Jones parameters of the bottom potential wall to attract the oligomer and solvent in question, thus mimicking a substrate. The top potential wall was placed far from the solution and made strongly attractive for the solvent, allowing for nonequilibrium diffusion of solvent through the film that would eventually condense at the top wall due to the attractive Lennard-Jones interactions. This method was refined by Negi, Lyulin, and Bobbert [30], who simulated thin-films of coarsegrained P3HT:PC $\mathbf{6 1}_{\mathbf{6}} \mathbf{B M}$ processed from different solvents. They allowed the solvent particles to diffuse through the film into a suspended solvent vapor above and would not remove solvent molecules until a certain density was reached in the top part of the box. The evaporation schemes themselves used in these papers hence have several advantages in terms of the physical processes included, but the time-scales needed to capture effects like nonequilibrium solvent diffusion through an oligomer thin-film are only reachable for very coarsegrained models. Furthermore, the substrate effects were not explicitly considered, and with the two described studies being the only ones that, to our knowledge, include these effects in solvent evaporation simulations, there is still room for improvement.

Three studies have been published in which substrate effects were not considered, but where alignment effects of molecules in a drying film were sought by using semiisotropic pressure coupling, i.e., only allowing the box to compress in the $z$-direction. Two of these relied on very coarse models [31,32], whereas a good compromise between chemical specificity and computational speed-up from coarse- graining was achieved using the MARTINI force-field [33] to coarse-grain a P3HT:PC ${ }_{61}$ BM system by Alessandri, Marrink, and co-workers [34]. In this, the rate of solvent removal was exponential with $1.25 \%$ per step to capture the effect of slower solvent evaporation with a decreasing solvent fraction, and the resulting morphologies were very convincing, which was underlined by the simulated diffraction patterns having distinct peaks for both the $\pi$ - and lamellar stacks close to the experimental ones, albeit hampered by implicit limitations of the coarse-grained bead types employed.

A few studies have more closely explored the structureproperty relationships in systems relevant for OPVs using atomistic molecular dynamics simulations with solvent evaporation [35-37]. Common for these was that no substrate effects were included and that isotropic pressure coupling was used during the solvent removal process. Although this evaporation method can give some insight into the bulk properties of the resulting dry materials, it is not expected to be fully representative for a thin-film.

In this work, we seek to devise a model for solution deposition of organic thin-films on substrates. This encompasses substrate effects, air-interface effects, and semi-isotropic pressure coupling in nonequilibrium, atomistic MD solvent evaporation simulations with no periodic boundary conditions in the $z$-direction. In addition to this model, we present here MD force fields for O-IDTBR and EH-IDTBR (see Fig. 1) as well as an analysis of the IDTBR thin-film nanostructure by combining GIWAXS experiments with the nonequilibrium MD solvent evaporation simulations. Furthermore, we present calculations of the electronic couplings between IDTBR molecules extracted from the MD simulations to analyze the structural pathways for electron transport in semicrystalline IDTBR domains, shedding light on their structure-property relationships.

\section{METHODS}

All molecular dynamics (MD) simulations were carried out in GROMACS 2018.3 [38] based on the OPLS-AA force field $[39,40]$. A robust and flexible script for solvent removal has been developed, allowing for a range of different removal schemes (from a suspended solvent vapor only, with a gradient through the system or randomly from the full extent of the system) and rates (linear and exponential) with separate control of the MD run parameters through a directory of configuration files (.mdp files). This script can be obtained from the authors upon request. This section is organized as a top-down description of the workflow, starting with the sample preparation and GIWAXS experiments in Sec. II A. Hereafter, the solvent evaporation simulations are described in Sec. II B, the substrate, air-interface, and solvent effects in Sec. II C, the force-field parametrization procedure for the IDTBR force fields in Sec. IID, and additional validation based on crystal simulations in Sec. II E.

\section{A. Sample preparation and GIWAXS measurements}

To compare the simulations to experimental data, thinfilms of O-IDTBR and EH-IDTBR (purchased from 1Material Inc.) were prepared on silicon wafers. The $\mathrm{Si}(100)$ 
substrates were initially cleaned for $30 \mathrm{~min}$ in an ultrasonic bath: $10 \mathrm{~min}$ in isopropanol, $10 \mathrm{~min}$ in acetone, and $10 \mathrm{~min}$ in demineralized water. The molecules, O-IDTBR and EHIDTBR, were dissolved in chloroform at a concentration of $30 \mathrm{mg} / \mathrm{mL}$ for $1 \mathrm{~h}$ on a $50{ }^{\circ} \mathrm{C}$ hot-plate with magnetic stirring. The solutions were then cooled down to room temperature before being spin-cast onto the substrates at $600 \mathrm{rpm}$ for $30 \mathrm{~s}$ and subsequently dried for ten minutes at $70{ }^{\circ} \mathrm{C}$. We furthermore fabricated a set of thin-films using the same procedure, which were additionally subject to annealing at $130{ }^{\circ} \mathrm{C}$ for $10 \mathrm{~min}$ in order to probe its effects on the thin-film properties. This dataset is presented in the SI, Fig. S4 [19]. The sample preparation procedure was identical to the one in Ref. [20], and the final film thicknesses are thus expected to be similar (280-290 nm).

All GIWAXS experiments were performed with an MPGenix.G06 microfocus X-ray source from Xenocs operated at $50 \mathrm{keV}$ and $1 \mathrm{~mA}$, monochromated to probe with a wavelength of $1.54 \AA(8.04 \mathrm{keV})$, and collimated with a set of pinholes downstream. The sample stage and image plate were kept in vacuum to avoid diffuse air scattering. A circular beam stop was placed just in front of the image plate. Alignment of the samples were carried out by using an X-ray eye camera while translating and rotating the sample stage. The incident angle was set to $0.18^{\circ}$ to maximize scattering from the thin-film and to avoid a signal from the silicon substrate. The distance from sample to detector was calibrated with Si powder to be $118.15 \mathrm{~mm}$.

\section{B. Solvent evaporation simulations}

The simulation box, having starting dimensions of $10 \times$ $10 \times 60 \mathrm{~nm}$, was initiated with a $\mathrm{SiO}_{2}$ substrate in the bottom (cf. Sec. II C) and a solution of 448 IDTBR molecules randomly distributed in preequilibrated chloroform on top of this (using gmx insert-molecules and gmx solvate). PBCs were only applied in the $x$ - and $y$-directions, whereas potential walls were applied in the $z$-direction at $z=0$ and $z=$ box $_{z}$ as described in Sec. IIC. The box was then equilibrated before initiating an exponential solvent evaporation process. For this, $2.5 \%$ of the remaining solvent was removed every step randomly throughout the extent of the box until reaching the near-linear regime (defined as removing less than $0.025 \%$ of the initial amount of solvent each step), whereafter the evaporation was continued linearly until a dry film was obtained. This amounts to a total of 133 steps. Both for the initial equilibration and for each step after solvent removal, the box was equilibrated for $0.2 \mathrm{~ns}$ in an $N V T$ ensemble and $2.0 \mathrm{~ns}$ in an NPT ensemble using the Berendsen barostat ( $\tau=2.0 \mathrm{ps}$ ) before running a $3.0 \mathrm{~ns}$ production run in the NPT ensemble using the Parrinello-Rahman barostat $(\tau=$ $12.0 \mathrm{ps}$ ), adding up to a total drying time of $0.7 \mu \mathrm{s}$. The $\mathrm{V}$ rescale thermostat ( $\tau=0.2 \mathrm{ps}$ ) and a leap-frog integrator with 2 fs steps were used for all runs. Furthermore, semi-isotropic pressure coupling of 1.0 bar was used with an isothermal compressibility of $4.5 \times 10^{-5} \mathrm{bar}^{-1}$ in the $z$-direction and $0.0 \mathrm{bar}^{-1}$ in the $x$ - and $y$-directions to facilitate shrinking of the box only in the $z$-direction to mimic the conditions in a drying thin-film. The particle mesh Ewald (PME) scheme was used to treat long-range electrostatics (a short-range cutoff of $1.2 \mathrm{~nm}$ ), whereas a cutoff of $1.2 \mathrm{~nm}$ was used for van der Waals (vdW) interactions. All hydrogens were constrained with the LINCS algorithm to reduce computational time.

Having obtained dry thin-films, these were subjected to a $100 \mathrm{~ns}$ simulated annealing cycle in an NPT ensemble: $10 \mathrm{~ns}$ with a linear temperature increase from 300 to $600 \mathrm{~K}, 70 \mathrm{~ns}$ at $600 \mathrm{~K}, 10 \mathrm{~ns}$ with a linear temperature decrease from 600 to $300 \mathrm{~K}$, and a final $10 \mathrm{~ns}$ at $300 \mathrm{~K}$. The resulting annealed

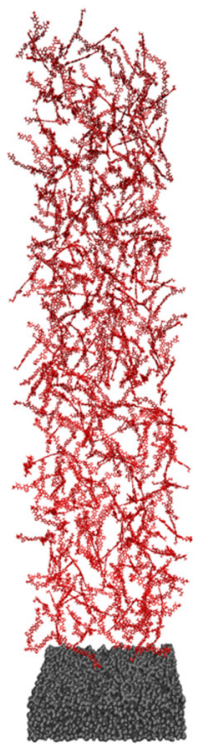

$0.00 \mu \mathrm{s}$

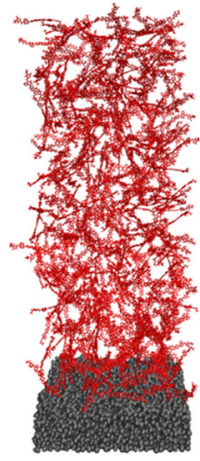

$0.16 \mu \mathrm{s}$

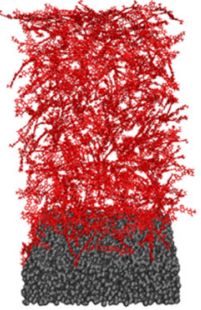

$0.31 \mu \mathrm{s}$

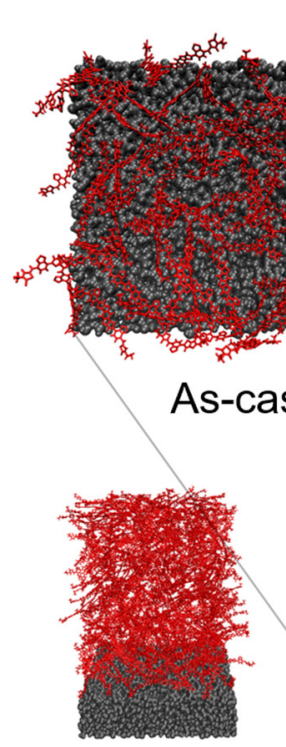

$0.47 \mu s$

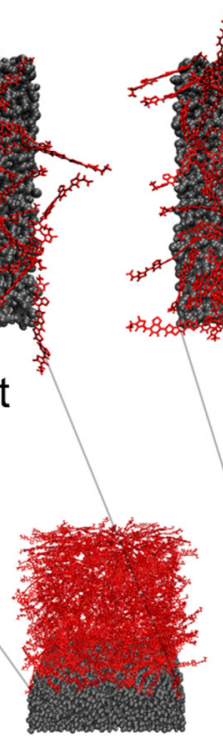

$0.67 \mu \mathrm{s}$

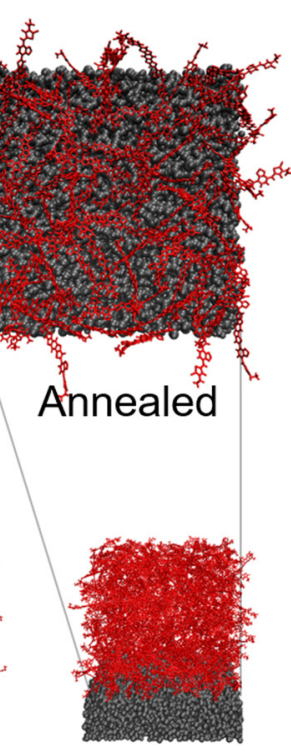

$0.77 \mu \mathrm{s}$

FIG. 2. Visualization of the solvent evaporation procedure for O-IDTBR (red) on an amorphous $\mathrm{SiO}_{2}$ substrate (gray). The final frames from five steps out of the total 133 steps are shown in addition to the annealed thin-film with their corresponding simulation times. Insets show the bottom layer of IDTBR molecules, i.e., molecules that are fully or partly within $4.5 \AA$ of the substrate, for the as-cast and annealed thin-films. Side chains and solvent molecules are not shown for clarity. 
thin-films, having a thickness of approximately $10 \mathrm{~nm}^{3}$, were then used for analysis in addition to the as-cast thin-films. The evaporation process is visualized in Fig. 2.

\section{Substrate, air-interface, and solvent interactions}

As we are seeking to validate the model using GIWAXS experiments, we have modeled the substrate using an explicit $\sim 2 \mathrm{~nm}$ layer of amorphous silicon dioxide $\left(\mathrm{SiO}_{2}\right)$ due to the thin-films being deposited on silicon wafers with native oxide (cf. Sec. II A). The parameters for $\mathrm{SiO}_{2}$ were based on the atomistic Clay force field [41] as adopted in Ref. [42], using exclusively nonbonded interactions between the silicon and oxygen atoms. The specific surface interactions between organic, conjugated molecules and $\mathrm{SiO}_{2}$ modeled using parameters from the Clay force field were validated by Roscioni et al. in a study of pentacene crystal growth [43]. We adapted the parameters to the OPLS-AA force field and initiated silicon and oxygen atoms randomly in a stoichiometric ratio 1:2 (5500 and 11000 atoms, respectively) in a $10 \times 10 \times 5 \mathrm{~nm}$ box without $\mathrm{PBC}$ in the $z$-direction using a strongly attractive bottom potential wall and a top potential wall with low interaction. The system was equilibrated in an NVT ensemble with the V-rescale thermostat at $600 \mathrm{~K}$ and subsequently cooled down to $300 \mathrm{~K}$ over the course of $1 \mathrm{~ns}$, resulting in a $\sim 2 \mathrm{~nm}$ layer of amorphous $\mathrm{SiO}_{2}$ aligned to the bottom of the box. This slab was used in the solvent evaporation simulations, held in place by an attractive interaction with a bottom potential wall of the 9-3 type in GROMACS, which is in essence a Lennard-Jones potential integrated over the volume behind the box boundary with an assigned density in atoms per $\mathrm{nm}^{3}$ of a given atom type. Here, a density of $49.94 \mathrm{~nm}^{-3}$, similar to the experimental value for bulk, crystalline silicon, is used. The atom type defining the interaction strength between the bottom wall and the $\mathrm{SiO}_{2}$ was the same as the silicon atom type used for the $\mathrm{SiO}_{2}$, but due to the potential walls in GROMACS only incorporating $\mathrm{vdW}$ interactions and not Coulombic interactions, the specific interaction was increased by a factor of $10^{3}$ to account for this (as a rule of thumb, ionic bonds are approximately a factor of $10^{3}$ times stronger than vdW forces). Any artefacts introduced by this are well shielded by the $\sim 2 \mathrm{~nm} \mathrm{SiO}_{2}$ slab that is dominated by ionic interactions, and the bottom potential wall will thus not interact with the solution deposited on the $\mathrm{SiO}_{2}$.

The air-interface in the solvent evaporation simulations was also modeled using a potential wall of the 9-3 type with an assigned density of $0.012 \mathrm{~nm}^{-3}$, corresponding to the experimental value for molecular nitrogen, $\mathrm{N}_{2}$, in ambient conditions with the standard OPLS-AA parameters for nitrogen defining the interaction strengths with the rest of the system. For both the top and bottom walls, the potentials were continued linearly within a distance of $1 \mathrm{~nm}$ from the box edges.

The solvent used for the sample preparation was chloroform, which was modeled using the OPLS-AA parameters by Caleman, van der Spoel, and co-workers [44]. To evaluate the solute-solvent interaction, the free energies of solvation (i.e., going from gas phase to solvation), $\Delta G_{\text {solv }}$, for $\mathbf{O}$ IDTBR and EH-IDTBR in chloroform were calculated using the Bennett's acceptance ratio (BAR) method and compared to corresponding simulations in two other commonly used solvents for IDTBR-based OPVs, namely chlorobenzene and $o$-xylene (both based on toluene OPLS-AA parameters also from Ref. [44]). The detailed simulation procedures can be found in the SI, Sec. S2.2 [19]. Additionally, solvation free energies in 1-octanol (based on standard OPLS-AA parameters) have been calculated in order to compare to a bad solvent for IDTBR. The results are listed in Table I. As is seen, chloroform and chlorobenzene are almost equally good solvents for IDTBR, and these two chlorinated solvents are better than the nonchlorinated $o$-xylene, reproducing the qualitative tendencies seen in experiments [45]. Here, the room-temperature solubility of O-IDTBR in chlorobenzene was found to be $45.8 \mathrm{mg} / \mathrm{mL}$, whereas it was found to be $18.9 \mathrm{mg} / \mathrm{mL}$ in $o$-xylene [45]; this corresponds to a difference in free energy of solvation of $2.2 \mathrm{~kJ} / \mathrm{mol}$, which is slightly lower than the $6.2 \pm 1.3 \mathrm{~kJ} / \mathrm{mol}$ predicted from the calculations. Estimating the O-IDTBR solubilities for chloroform and 1-octanol using a relative measure from the solubility in chlorobenzene yields values of around $87 \mathrm{mg} / \mathrm{mL}$ for chloroform and $10^{-2} \mathrm{mg} / \mathrm{mL}$ for 1-octanol (i.e., practically insoluble, as expected). Note that the less negative $\Delta G_{\text {solv }}$ values for EH-IDTBR compared to O-IDTBR do not necessarily equal lower solubility, as the thermodynamic equilibrium between the solid-state EHIDTBR and a given solvent is different from that of solid-state O-IDTBR and the same solvent.

We note that the present simulations do not include mechanical shear stress effects, although these are expected to be relevant for spin-coating deposition. It has previously been found in a combined experimental and atomistic MD study that medium or high shear rates (corresponding to spin-coating speeds of several thousand rpm) can promote crystallinity in organic thin-films, but that low shear rates did not promote crystallinity significantly [46]. Another MD study employing very coarse-grained models of an OPV donor:Acceptor blend found that high shear rates could promote phase separation and increase crystallinity of the acceptor domains, but again that low shear rates did not have a significant effect compared to simulations not including shear stress [32]. Although the simulations herein are thus a truer representative of drop-casting than they are of spin-coating due to the lack of inclusion of shear stress, the very slow spincoating speeds employed for the sample fabrication (600 rpm)

TABLE I. Calculated free energies of solvation in different solvents (300 K, 1 bar) for the presented models of O-IDTBR and EH-IDTBR.

\begin{tabular}{lcccc}
\hline \hline$\Delta G_{\text {solv. }}(\mathrm{kJ} / \mathrm{mol})$ & Chloroform & Chlorobenzene & $o$-xylene & 1 -octanol \\
\hline O-IDTBR & $-357.3 \pm 0.9$ & $-355.7 \pm 0.9$ & $-349.5 \pm 0.8$ & $-330.0 \pm 0.9$ \\
EH-IDTBR & $-326.8 \pm 0.2$ & $-319.8 \pm 0.8$ & $-312.5 \pm 0.6$ & $-301.4 \pm 2.1$ \\
\hline \hline
\end{tabular}


induces only low mechanical shear stress in the thin-films and ensures better comparability than for samples spun at the usual speeds of $\sim 2000 \mathrm{rpm}$. Work on including these effects in future simulations is ongoing.

\section{IDTBR force-field parametrization}

The force fields for IDTBR nonfullerene acceptors developed herein were built in the framework of OPLS-AA with parameters based on density functional theory (DFT) calculations carried out in GAUSSIAN 16 [47]. For these, we employed the extensively used global hybrid functional B3LYP [48-50], which incorporates $20 \%$ exact exchange, as well as the $\omega$ B97X-D functional [51,52], which is a highly parametrized, range-separated functional incorporating $100 \%$ exact exchange at long range. In combination with the empirical atom-atom dispersion correction (denoted by D), $\omega \mathrm{B} 97 \mathrm{X}-\mathrm{D}$ is highly accurate in describing thermochemistry and nonbonded interactions [52]. Throughout, we have used the Pople style basis-set 6-311++G(d,p) [53,54], a triple- $\zeta$ basis with diffuse and polarization functions on both heavy atoms (nonhydrogens) and hydrogens to capture the relevant effects of all nonbonded interactions. Using these quantum chemical calculations, we carefully parametrized the potentials involving atom types that were not present in OPLS-AA as well as all torsional potentials, the exact procedures of which are listed in the SI, Sec. S2.1, along with all resulting nonstandard OPLS-AA parameters used [19,55].

Summarizing, the atomic partial charges were assigned based on symmetrized ESP (ChelpG) charges of the minimum energy conformation of a methyl-substituted IDTBR optimized at the B3LYP/6-311++G(d,p) level of theory, and the atom types were assigned based on the existing OPLSAA atom types and renamed copies of these (see the SI, Fig. S1 and Table S1) [19], taking great care to capture the asymmetry around the $s p^{3}$-hybridized linking carbon in the indacenodithiophene (IDT) subunit (we refer to the discussion in the SI, Sec. S2.1) [19]. Bond-type parameters were mainly adopted from OPLS-AA, and the backbone equilibrium angles were in most cases determined from the B3LYP/6-311++G(d,p) optimized minimum energy conformation, whereas the side-chain equilibrium angles and the force constants for both backbone and side-chain angles were adopted from OPLS-AA. Most of the proper dihedrals in the IDTBR backbone are modeled by the standard OPLSAA Ryckaert-Bellemans (RB) parameters for aromatic systems, whereas for the side chains they are modeled by the standard OPLS-AA RB parameters for alkanes. However, and most importantly, dihedral parameters for the torsional potentials of the linking bonds between rhodanine $(\mathrm{RH})$ and benzothiadiazole (BT), between IDT and BT, and between IDT and the side chains were determined through an iterative Boltzmann inversion (IBI) procedure, resulting in coefficients for Ryckaert-Bellemans functional forms that, when included in the remainder of the force field, reproduce the $\omega \mathrm{B} 97 \mathrm{X}$ $\mathrm{D} / 6-311++\mathrm{G}(\mathrm{d}, \mathrm{p})$ torsional potentials. Using this specific DFT level of theory is consistent with the recommendations in the most recent publication on the reparametrization of OPLS-AA peptide backbone torsions [56]. Our IBI procedure was performed as follows:

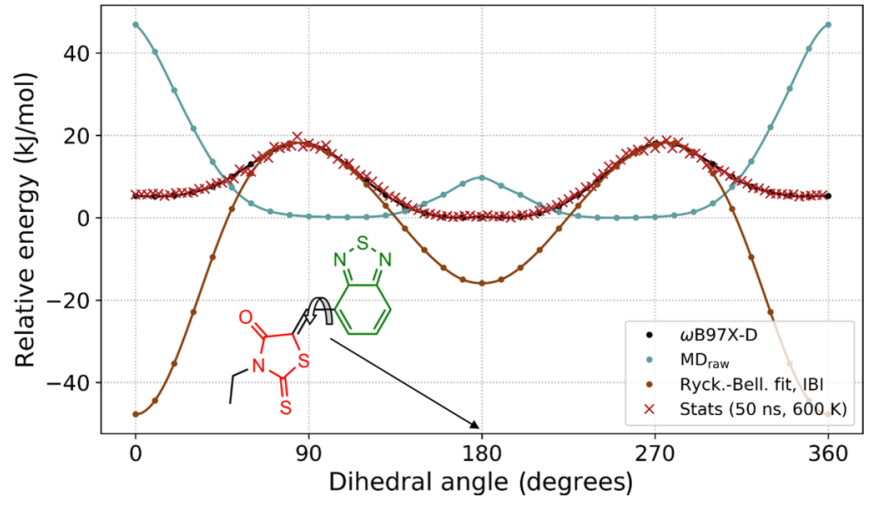

FIG. 3. Visualization of the IBI fitting procedure employed to mimic the $\omega \mathrm{B} 97 \mathrm{X}-\mathrm{D}$ potentials for the rhodanine-benzothiadiazole (RH-BT) torsion using Ryckaert-Bellemans functions to parametrize the force field.

(a) DFT relaxed scan at the $\omega \mathrm{B} 97 \mathrm{X}-\mathrm{D} / 6-311++\mathrm{G}(\mathrm{d}, \mathrm{p})$ level of theory (i.e., geometry optimizations while constraining only the relevant torsional angle) of a methyl-substituted IDTBR in vacuum.

(b) MD relaxed scan (with a convergence criterion of a maximum force of $10 \mathrm{~kJ} \mathrm{~mol}^{-1} \mathrm{~nm}^{-1}$ ) of the torsional angle of reduced systems (i.e., RH-BT for the RH-BT torsion, 4,4,9,9-tetramethyl-IDT-BT for the IDT-BT torsion, and 4,4diethyl-9,9-dimethyl-IDT for the IDT-to-side-chain torsions) with IDTBR partial charges using our parametrized version of the OPLS-AA force field with the potentials of the four dihedral quadruplets in question set to zero.

(c) Nonlinear least-squares fitting of a Ryckaert-Bellemans functional form [57] [Eq. (1)] to the difference between the DFT scan and the MD scan:

$$
V_{\mathrm{RB}}\left(\phi_{i j k l}\right)=\sum_{n=0}^{5} C_{n} \cos ^{n}(\psi) \quad \text { with } \quad \psi=\phi-180^{\circ},
$$

where $\phi_{i j k l}$ is the dihedral angle of a quadruplet in degrees. This provided the initial RB parameter guesses.

(d) Long vacuum simulation (10-50 ns depending on the iteration) in an $N V T$-ensemble at $600 \mathrm{~K}$ using $1 \mathrm{fs}$ steps and subsequent collection of torsion statistics (using gmx angle).

(e) Boltzmann inversion of the statistics and fitting of RB parameters to the difference between the resulting potential and the $\omega \mathrm{B} 97 \mathrm{X}-\mathrm{D}$ potential-these RB parameters are then linearly combined with the ones from the previous step.

(f) Iterations of steps (d) and (e) until convergence [which is defined by a root-mean-square deviation (RMSD) of $<1.0 \mathrm{~kJ} / \mathrm{mol}$ for the MD statistics to the DFT potential].

The resulting Boltzmann inverted statistics are visualized in Fig. 3 for the RH-BT torsion, and in the SI, Figs. S2 and S3, for the IDT-BT and the IDT-to-side-chain torsions, respectively [19], and the optimized RB parameters for these torsions are listed in the SI, Table S2 [19].

\section{E. Crystal simulations and validation}

Recently, crystal structures based on x-ray diffraction measurements of single crystals grown by antisolvent vapor diffusion were published for O-IDTBR and EH-IDTBR [22]. As 
it has previously been shown that O-IDTBR has a crystallization transition at $T_{c}=115^{\circ} \mathrm{C}$ [20], a high structural order can potentially be induced locally in annealed thin-films of O-IDTBR, whereas this is not the case for EH-IDTBR (cf. the SI, Sec. S3) [19]. We have thus chosen to include simulations based on these crystal structures in order to investigate the differences in both structure and properties between ordered, crystalline domains and more disordered domains in the thin-films. The crystal simulations were performed using the same force fields and run parameters as for the thin-films but with full periodic boundary conditions and anisotropic pressure coupling (i.e., with no considerations of interface effects), with isothermal compressibilities of $4.5 \times 10^{-5} \mathrm{bar}^{-1}$ in all directions.

The crystal simulations additionally allowed for further validation of the force fields. First, the RMSD between the experimental crystal structures and the corresponding energy-minimized structures (to maximum interatomic forces $<10 \mathrm{~kJ} \mathrm{~mol}^{-1} \mathrm{~nm}^{-1}$ ) using the IDTBR force fields was calculated. This yielded values of only 0.17 and $0.31 \AA$ for O-IDTBR and EH-IDTBR, respectively, demonstrating that especially the bonded parameters of the force field very accurately reproduce the experimental molecular geometries. Secondly, the crystal lattice parameters $(a, b, c)$ of $173 \mathrm{~K}$ $N P T$ equilibrated boxes were compared to the experimental ones (obtained at $173 \mathrm{~K}$ ) in order to get an impression of how well the nonbonded parameters of the force field were able to describe the intermolecular interactions and hence the crystal packing. Relative deviations of $2.9 \%,-1.1 \%$, and $0.4 \%$ were found for $(a, b, c)$ of the O-IDTBR crystal simulation, whereas deviations of $-3.6 \%, 5.0 \%$, and $0.0 \%$ were found for EH-IDTBR. For O-IDTBR, the deviation of $2.9 \%$ along $a$ corresponds to an elongation in the $\pi$-stacking direction, whereas the deviations of $-3.6 \%$ and $5.0 \%$ along $a$ and $b$ for EH-IDTBR can collectively be seen as a slide of the $\pi$-stacks. All of these deviations are, however, minor, which demonstrates that the nonbonded parameters are indeed able to describe the intermolecular interactions reasonably well.

\section{RESULTS AND DISCUSSIONS}

The results presented are based on O-IDTBR and EHIDTBR thin-films cast from chloroform onto an amorphous $\mathrm{SiO}_{2}$ substrate through $0.7 \mu$ s atomistic MD solvent evaporation simulations as well as on their $0.1 \mu$ s annealed versions (adding up to a total simulation time of $0.8 \mu \mathrm{s}$ ). The detailed simulation procedures can be found in Sec. II B. These are compared to results from GIWAXS measurements of $\mathbf{O}$ IDTBR and EH-IDTBR thin-films spin-cast from chloroform onto silicon wafers (cf. Fig. 4). The detailed sample preparation procedures can be found in Sec. II A. Furthermore, MD simulations based on the experimentally obtained O-IDTBR and EH-IDTBR crystal structures [22] are presented to be able to compare the results from the thin-film simulations to corresponding results from perfectly ordered systems.

To reduce computational cost, the simulations were initiated at an IDTBR concentration of approximately 200 $\mathrm{mg} / \mathrm{mL}$, which is an order of magnitude higher than the initial concentration of the solutions used in experiments. This was, however, deemed to be sufficiently dilute, having

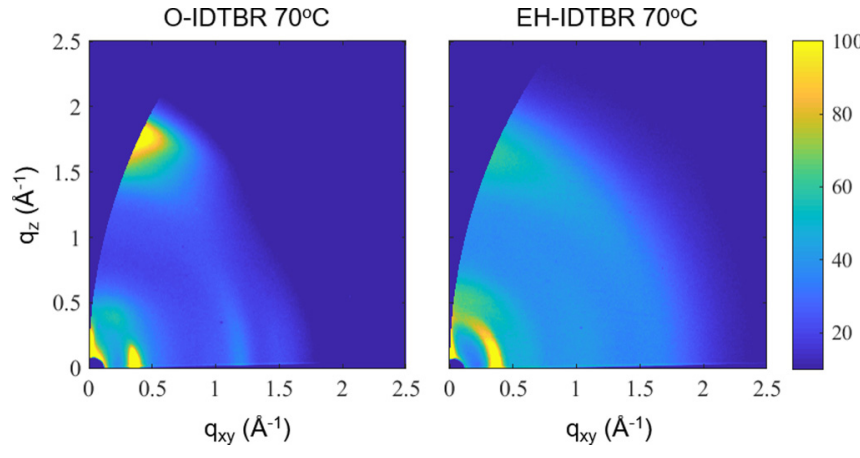

FIG. 4. 2D GIWAXS data of the O-IDTBR (left) and EHIDTBR (right) thin-films cast on silicon substrates and dried at $70^{\circ} \mathrm{C}$.

a low number of close interactions $(<8 \AA)$ of less than $5 \%$ relative to the dry films. The dry, simulated thin-films have a thickness of $\sim 10 \mathrm{~nm}$, which is an order of magnitude thinner than the experimental samples. Again, this is necessary to keep the computational cost at a feasible level. As the nanostructural characteristics such as $\pi$-stacking and relative alignment to the substrate occur at length-scales at least an order of magnitude lower than the extent of the system, these are expected to be statistically representative, whereas the system size is only sufficient to extract qualitative tendencies of longer-range order.

\section{A. Structural properties}

In Fig. 4, GIWAXS images of O-IDTBR and EH-IDTBR thin-films on silicon wafers are shown. From the sharper peak features in the O-IDTBR image, it can qualitatively be deduced that O-IDTBR has a higher degree of order than EHIDTBR in thin-films. Looking at the O-IDTBR data, a broad out-of-plane peak can be observed at $q_{z} \sim 1.8 \AA^{-1}$, indicating $\pi$-stacking with a face-on orientation to the substrate. For the in-plane direction, a sharp feature at $q_{x y} \sim 0.4 \AA^{-1}$ indicates a longer-range order being present. This feature is unlikely to be directly related to the length of the side chains as usually seen for lamellar stacks in polymeric thin-films with side chains attached to $s p^{2}$-hybridized carbons. Instead, IDTBR molecules employ $s p^{3}$-hybridized linking carbons, to each of which two side chains are bonded [cf. (1)]. These side chains thus have a preferential conformation that is normal to the plane of the backbone, i.e., pointing away from each other, which induces bulkiness around the central IDT unit. This, in turn, means that the $\pi$-stacking is expected to occur between the terminal RH units and/or the bridging BT units in the thin-films, and that the in-plane feature could be related to distances between "columns" of these $\pi$-stacks. Similar, but less defined, features are present for EH-IDTBR thinfilms. These are analyzed in detail both qualitatively and quantitatively below.

As the basis of the structural analysis of the simulations, the center of mass (COM) of each of the conjugated ringsystems was defined as distance evaluation points: One for each of the RH units, one for each of the BT units, one for each of the thiophenes in the IDT unit, and one for the central benzene in the IDT unit, amounting to a total of seven evaluation points per molecule (cf. Fig. 1). The normal vectors 

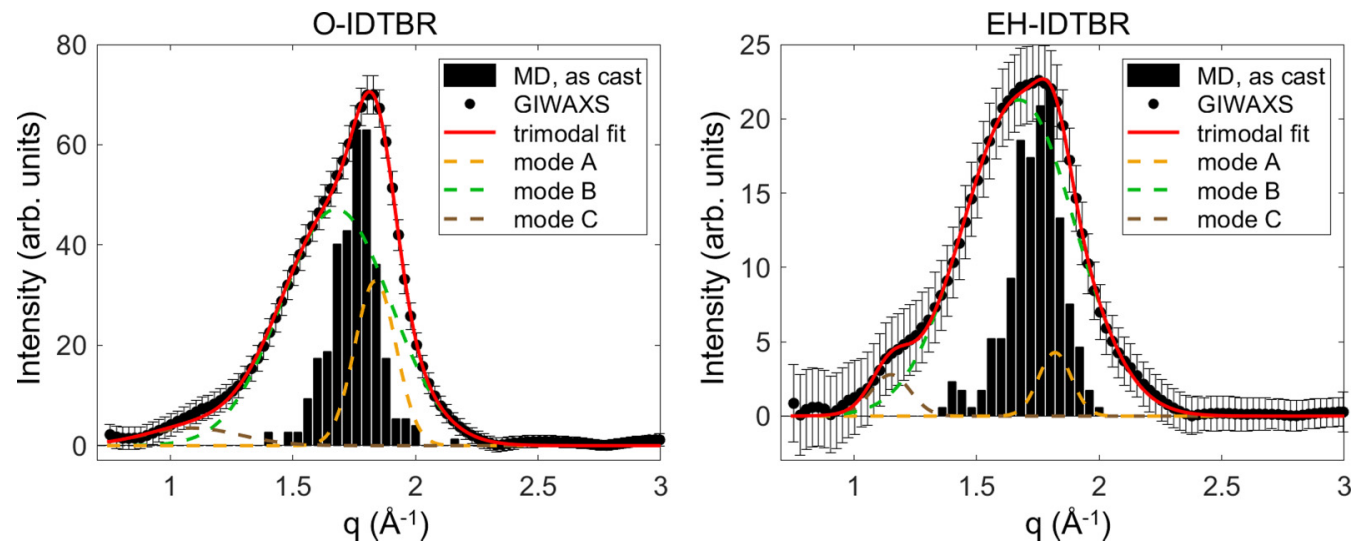

FIG. 5. Azimuthal integrations (black dots) and corresponding fits (red lines) to the (010) $\pi$-stack peaks of the O-IDTBR and EH-IDTBR thin-films cast at $70{ }^{\circ} \mathrm{C}$ compared to the $\pi$-stacking distances extracted from the as-cast simulated thin-films (gray bars).

of the individual planes spanned by these seven ring systems were also computed to be able to evaluate the relative orientation of the molecular pairs. To quantify the $\pi$-stacking distance in the simulations, the distance from each evaluation point in each molecule to all evaluation points in all other molecules were computed, and the shortest interaction for each molecule was extracted (see the SI, Fig. S11 for representative images of $\pi$-stacking molecular pairs) [19]. These were then subject to a filtering based on an evaluation of relative alignment using a cutoff of $\arccos (0.9) \sim 25.8$ degrees from perfectly (anti)parallel alignment to define cofaciality (only a few molecular pairs were disqualified from this). For the cofacial pairs, the average of the distance from the relevant evaluation point of molecule $A$ to the plane of the relevant ring-system of molecule $B$ and the distance from the relevant evaluation point of molecule $B$ to the plane of the relevant ring-system of molecule $\mathrm{A}$ was defined as the $\pi$ stacking distance to account for cases in which the $\pi$-stacking ring-systems were slightly offset with respect to each other (as the direct evaluation point to evaluation point distance would in this case be an overestimation of the actual $\pi-\pi$ distance). Histograms of the resulting $\pi$-stacking distances for all cofacial, closely interacting molecular pairs in the as-cast thin-film simulations are plotted in Fig. 5 with the corresponding GIWAXS data for the low-temperature cast $\left(70{ }^{\circ} \mathrm{C}\right.$ ) thin-films. The real-space $\pi$-stacking distances for all simulations (as-cast and annealed thin-films as well as the crystal structures) and the values extracted from the GIWAXS data for the low-temperature cast thin-films are presented in Table II.

As seen in Fig. 5, the simulated $\pi$-stacking distances are in excellent correspondence with the GIWAXS data for both
O-IDTBR and EH-IDTBR in terms of the maximum intensity positions for the high- $q$ peak at $q_{z}$. Further analysis of the GIWAXS results shows that trimodal GAUSSIAN functions fit the data very precisely: Two high-intensity modes in the high- $q$ range, which are associated with the $\pi$-stacking, and a low-intensity tail mode in the lower- $q$ range, which could be ascribed to packing of side chains. The mean values and the corresponding errors of the fits of the former two, mode A and mode B, are listed in Table II. This multimodality is not unambiguously present in the histograms of the simulation data, although some indications of shoulders toward the lower- $q$ range of the histograms can be seen. The resolution of this effect in the thin-film simulations is most probably limited by statistics as only 448 molecules are included in these, and the simulation values listed in Table II are hence only based on single GAUSSIAN fits. However, manual inspection of the molecular pairs from the simulations suggests that the bulkiness of the terminal ethyl group on the RH unit is the reason for the bimodality of the $\pi$-stacking observed in experiments: If the ethyl group points inward in a $\pi$-stack including a RH unit, the stack is slightly distorted and elongated, while a similar stack but with the ethyl group pointing outward will have a closer interaction. As seen in Fig. 6 showing two representative $\pi$-stacking pairs from the simulations, the distances are furthermore very close to the ones identified in the GIWAXS analysis for mode A and mode B. A future molecular design of IDTBR derivatives should hence include fully planar terminal units instead of the ethyl substituted $\mathrm{RH}$ unit in order to promote shorter $\pi$-stacking distances and thus stronger intermolecular electronic coupling (cf. Sec. III C).

Looking at the $\pi$-stacking distances in Table II, no significant differences between O-IDTBR and EH-IDTBR can

TABLE II. Mean and standard deviation in $\AA$ of GAUSSIANS fitted to the $\pi$-stacking distances from the simulations compared to the $\pi$-stacking distances and the corresponding errors extracted from GIWAXS measurements (modes A and B from multimodal Gaussian fits; cf. Fig. 5).

\begin{tabular}{lccccr}
\hline \hline & & MD simulations & & \multicolumn{2}{c}{ GIWAXS } \\
\cline { 2 - 4 }$\pi$-stack $(\AA)$ & as-cast & anneal. & crystal & & mode A \\
\hline O-IDTBR & $3.60 \pm 0.22$ & $3.68 \pm 0.25$ & $3.52 \pm 0.09$ & $3.43 \pm 0.02$ & $3.76 \pm 0.01$ \\
EH-IDTBR & $3.64 \pm 0.25$ & $3.68 \pm 0.26$ & $3.51 \pm 0.12$ & $3.45 \pm 0.03$ & $3.76 \pm 0.01$ \\
\hline \hline
\end{tabular}




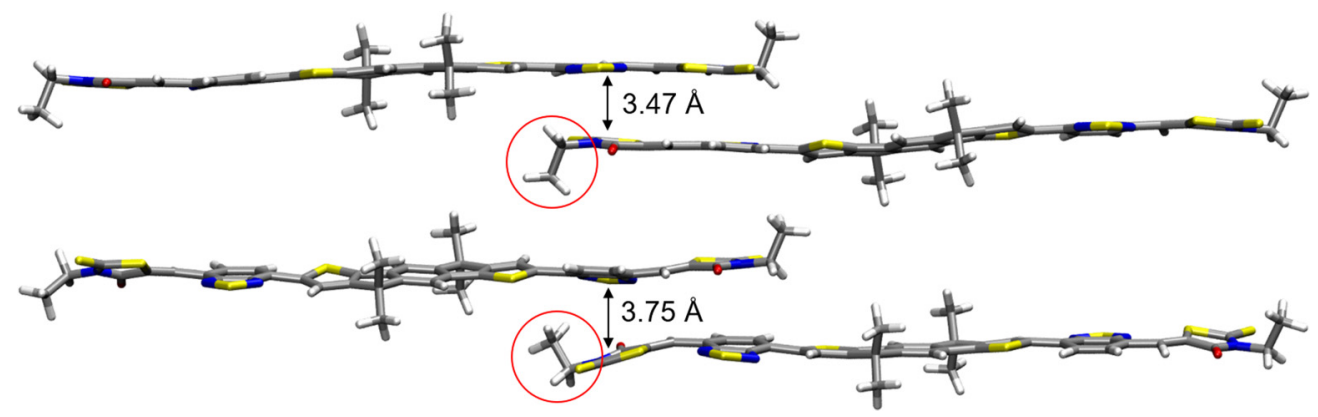

FIG. 6. Representative molecular pairs from EH-IDTBR crystal simulations at $300 \mathrm{~K}$ illustrating the bimodality of the $\pi$-stacks caused by the terminal ethyl group on the RH units (circled in red). The $\pi$-stacking distances for the two specific pairs as calculated with the method described in Sec. III A are indicated. The side chains on IDT are substituted for methyl groups for clarity (only in the visualization).

be seen. The distances increase slightly in the thin-film simulations upon annealing from $3.60 \pm 0.22 \AA$ the as-cast $\mathbf{O}$ IDTBR thin-film to $3.68 \pm 0.25 \AA$ for the annealed and from $3.64 \pm 0.25 \AA$ the as-cast EH-IDTBR thin-film to $3.68 \pm$ $0.26 \AA$ for the annealed. These are all in good agreement with the GIWAXS data, spanning the range of both modes from the measurements. The simulations of the ordered crystals logically pack closer with lower standard deviations, namely $3.52 \pm 0.09 \AA$ for O-IDTBR and $3.51 \pm 0.12 \AA$ for EHIDTBR. In conclusion, the short-range structural properties are very satisfactorily modeled using the solvent evaporation simulation procedure devised herein.

Regarding longer-range order, the GIWAXS measurements on the low-temperature cast thin-films showed intense peaks in $q_{x y}$ with values of $17.57 \pm 0.02$ and $17.08 \pm 0.05 \AA$ based on fits using the Voigt model for O-IDTBR and EHIDTBR, respectively, whereas the corresponding numbers for the $130{ }^{\circ} \mathrm{C}$ annealed samples were $14.40 \pm 0.02$ and $16.49 \pm 0.40 \AA$ (see the SI, Fig. S6) [19]. Regrettably, this longer-range order was not present to a quantifiable degree in the thin-film simulations (simulated scattering signals were very weak; cf. the SI, Fig. S7) [19,58]. However, it can be seen from radial distribution functions of the IDTBR COMs (note: The molecular COMs, not the residual COMs corresponding to the individual evaluation points) that some of the characteristic distances from the crystal simulations coincide with the more probable distances from the thin-film simulations (see Fig. 7). The O-IDTBR as-cast thin-film exhibits a broad distribution from 10 to $20 \AA$, which upon annealing sharpens to a bump with a maximum probability density at $14.5 \AA$ with a weak shoulder at around 17.2 $\AA$. The former of these is also present as a peak in the crystal simulations, corresponding to an intermolecular distance across the side-chain-filled space in the plane perpendicular to the $\pi$-stacking plane (cf. red arrows on the left panel of Fig. 7). This could thus have the necessary contrast to show up in scattering measurements, and it indeed corresponds very well to the strong peak at $14.40 \AA$ found for the $130{ }^{\circ} \mathrm{C}$ annealed O-IDTBR thin-film.

Looking at the EH-IDTBR thin-film simulations, the maximum probability density moves from 12.4 to $13.0 \AA$ upon annealing, and the shoulder at around $17.0 \AA$ gets more pronounced. The distances are also present as intense peaks in the crystal simulation, and they both show up in the GIWAXS spectrum, the former, however, with a low intensity. An inspection of the crystal structure shows that the latter indeed seems to have more contrast, i.e., extending across a sidechain-filled space, and it occurs in the plane perpendicular to the $\pi$-stacking plane (cf. red and blue arrows in the right panel of Fig. 7).

In summary, these results indicate that the simulated annealing has, although to a low degree, increased the structural order for the low- $q$ range in the thin-film simulations. It remains, however, that the reasonably accessible simulation times for atomistic MD are not sufficient to induce a high structural order in the low- $q$ range from randomly initiated simulations of solution deposited small-molecule systems.

\section{B. Effects of the substrate}

As described in Sec. II C, the parameters used for the amorphous $\mathrm{SiO}_{2}$ substrate have previously been validated in terms of their interaction with organic molecules in vapor deposition simulations [43]. To further validate these in the context of solvent evaporation simulations, we have compared the relative orientation of the molecules and the substrate plane in the simulations to the experimental $\pi$-stack orientation extracted from the (010) peak from the GIWAXS measurements, and we plotted these in Fig. 8. Each of the GIWAXS data points represents the intensity of a fixed- $q$ integration over a $2^{\circ}$ wedge in $\omega$ of the spectra shown in Fig. 4 using an analysis similar to the one visualized in Fig. 5. These fits are shown in the SI, Figs. S8 and S9 [19]. The simulation data points are obtained as the angles between the normal vector of the substrate and the normal vectors of the planes spanned by each of the seven evaluation point ring-systems in the IDTBR molecules.

Although the relative orientations from the simulations show a quantitative deviation from the corresponding GIWAXS data, the qualitative behaviors are very similar: A face-on stacking is favored for both thin-films, i.e., highest intensities at low $\omega$-values, with a tendency for O-IDTBR to be more textured than EH-IDTBR, i.e., having a sharper orientation profile. Regarding the quantitative deviation, it is important to note that whereas the GIWAXS intensity depends strongly on the local order of several molecules and will thus not yield a signal for a disordered region, all molecules have equal weight in the analysis of the simulated thinfilms regardless of the order of their surrounding molecules. This can explain the less sharp decrease of the signal from the simulated thin-films with higher angles compared to the 

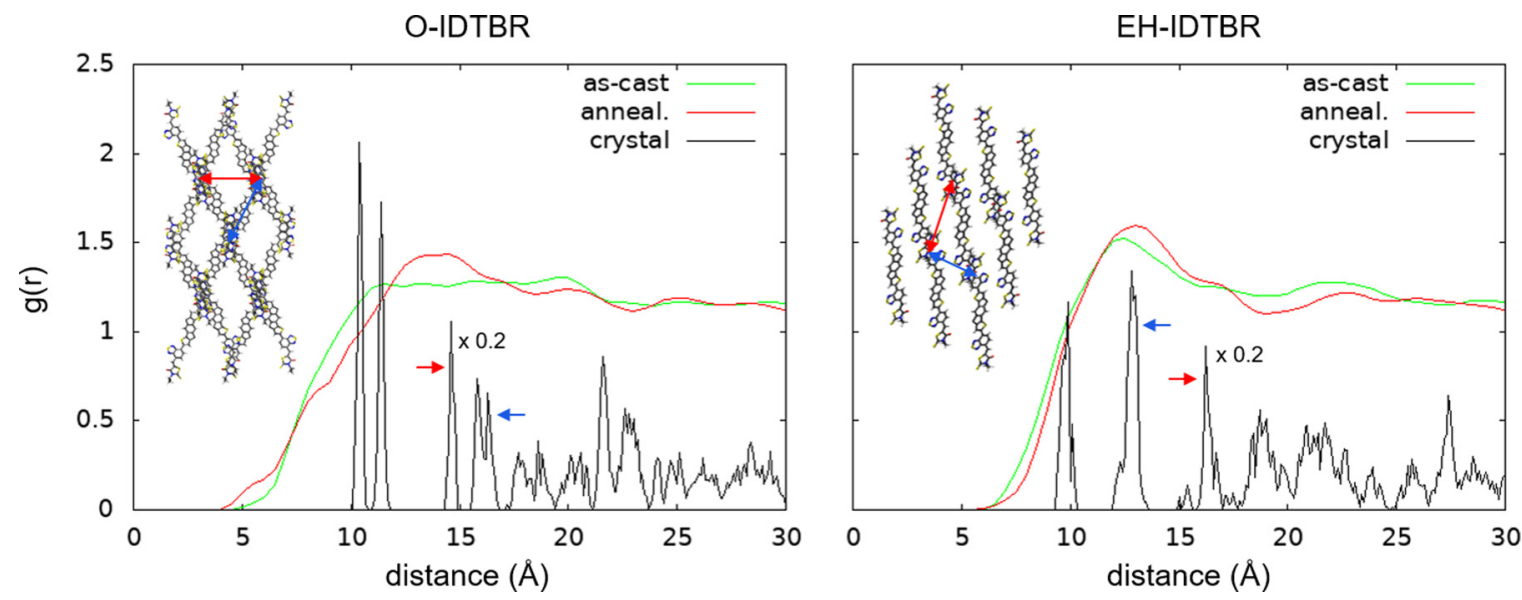

FIG. 7. Radial distribution functions of the COM positions in the simulations of O-IDTBR (left) and EH-IDTBR (right) as-cast thin-films (green; Bezier spline fit), annealed thin-films (red; Bezier spline fit), and crystals (black; scaled by a factor of 0.2). Insets are top views ( $\pi$-stacks in the out-of-plane direction) of the ideal crystal structures without side chains to ease the view; red arrows mark the distances present as peaks in the GIWAXS spectra of the annealed thin-films, whereas blue arrows mark distances that do not show up in these GIWAXS spectra.

measurements. Summarizing, the substrate effects of amorphous $\mathrm{SiO}_{2}$ are satisfactorily modeled with the employed parameters in the context of this study. We note, however, that functional devices such as OPVs or OFETs are most often deposited directly on top of organic layers of polymers or small molecules that act as charge collection layers between the active layer and the solid-state oxide or metal electrodes. Surface properties such as the hydrophobicity of these layers can vary significantly from that of amorphous $\mathrm{SiO}_{2}$, which affects the thin-film growth [59]. In this study, a simple setup with few components was prioritized to enable a more direct comparison between simulations and experiments, but future

$\pi$-stack orientation

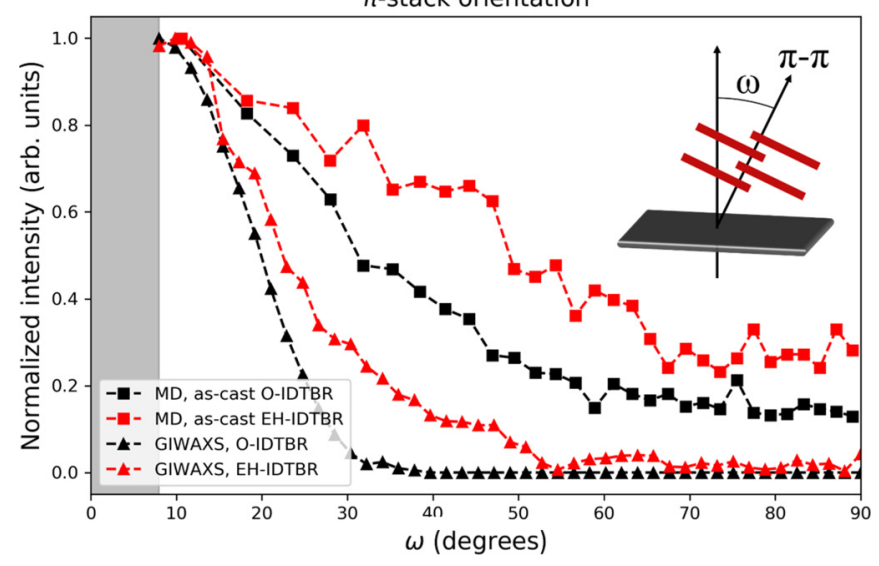

FIG. 8. Orientational distribution of the $\pi$-stacks relative to the substrate (cf. the inset) obtained from the (010) GIWAXS-peak for O-IDTBR and EH-IDTBR low-temperature cast thin-films (black and red squares, respectively) compared to the corresponding MD distributions computed as the angles between the normal vector of the substrate and those of each of the evaluation point ring-systems in the as-cast thin-films (black and red triangles, respectively). The gray-shaded area from $0^{\circ}$ to $8^{\circ}$ represents the range of angles not probed due to the fixed grazing incidence angle. studies should thus focus on implementing substrates that are more directly relevant for functional devices.

\section{Electronic properties}

To determine if the structural characteristics discussed are indeed beneficial to the transport properties, we extracted the unique molecular pairs from each of the simulated O-IDTBR and EH-IDTBR as-cast and annealed thin-films to perform electronic structure calculations on them. To reduce computational costs, the side chains were substituted by methyl groups, a procedure that is justified by the insulating nature of alkyl side chains as well as the negligible impact of their length/size on the backbone electronic structure [60]. We then did single-point DFT calculations at the B3LYP/6-31G(d) level of theory on the pairs and each of the molecules that the pairs consist of, and afterward we calculated the electronic couplings from their frontier orbitals [highest occupied molecular orbital (HOMO); lowest unoccupied molecular orbital (LUMO)] using the projective method [61,62], which is valid for intermolecular coupling of identical molecules. The use of B3LYP/6-31G(d) is justified as a compromise between computational cost and accuracy in terms of the description of frontier orbital properties (see below). Note that incorporating diffuse functions in the basis set for the single-point calculations of the pairs would lead to an overcomplete basis because diffuse functions centered on one molecule would overlap with the other molecule in the pair, thus inhibiting the use of the projective method by causing the orthogonalization of the overlap matrices to break down.

To assess the description of the frontier orbitals at the chosen level of theory, B3LYP/6-31G(d), the HOMO and LUMO energies were computed for each molecule in the extracted pairs used for the coupling calculations and plotted in the left panel of Fig. 9; in the right panel, frontier orbitals of a B3LYP/6-31G optimized, methyl-substituted IDTBR are visualized. Although a direct comparison of calculated LUMO energies in vacuum to electrochemical measurements in solvent of electron affinity is not valid, the calculated HOMO 


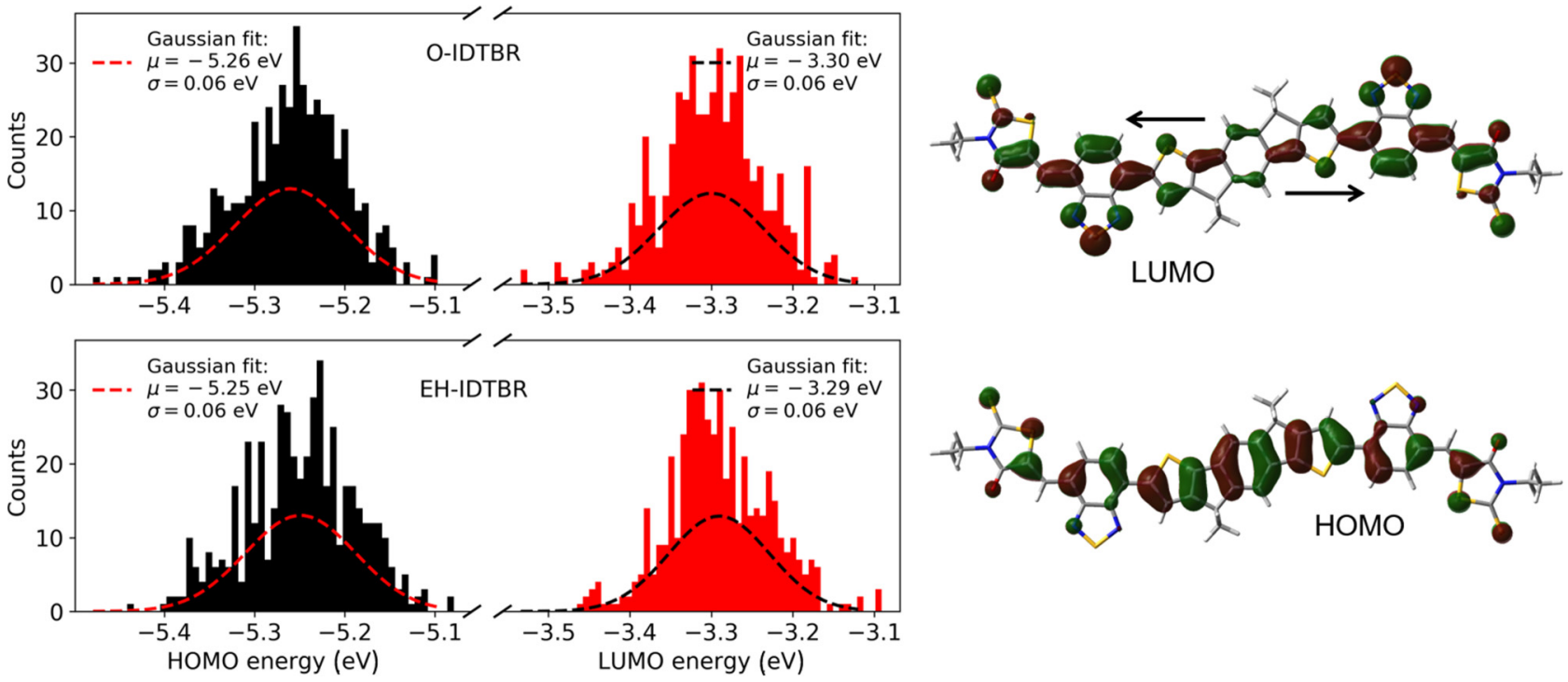

FIG. 9. Left: Frontier orbital energies of the 500 molecules from the 250 most closely stacked pairs in each of the simulated O-IDTBR and EH-IDTBR as-cast thin-films on $\mathrm{SiO}_{2}$ substrates. The arithmetic mean, $\mu$, and standard deviation, $\sigma$, of a GAUSSIAN fit to each of the data-sets are indicated. Right: Frontier orbitals of a B3LYP/6-31G(d) optimized, methyl substituted IDTBR molecule. Arrows mark the directions of the change in electron density when going from HOMO to LUMO. An isovalue (contour threshold) of 0.02 au is used in the visualization.

energies can serve as a good estimate of the ionization potential for high-throughput calculations. For both O-IDTBR and EH-IDTBR, the HOMO and LUMO energies were centered around -5.25 and $-3.29 \mathrm{eV}$, leading to a HOMO-LUMO gap of $1.96 \mathrm{eV}$, which is close to the experimental $\lambda_{\max }$ of uv-vis thin-film absorptions of 1.80 and $1.84 \mathrm{eV}$, respectively [20]. Experimentally, the O-IDTBR and EH-IDTBR as-cast thin-film electron affinities were determined to be -3.88 and $-3.90 \mathrm{eV}$, whereas the ionization potentials were estimated to be -5.51 and $-5.58 \mathrm{eV}$ based on optical gaps of 1.63 and $1.68 \mathrm{eV}$, respectively [20]. The correspondence of the calculated values to these are decent. To test the dependence of the basis set, the calculations were repeated for an optimized, methyl-substituted IDTBR molecule at the B3LYP/6-31G(d) and B3LYP $/ 6-311++\mathrm{G}(\mathrm{d}, \mathrm{p})$ levels of theory. The significant increase in number of basis functions (from 996 to 1668) yielded HOMO and LUMO energies of -5.53 and $-3.47 \mathrm{eV}$, respectively, which are improvements compared to the -5.28 and $-3.17 \mathrm{eV}$ of the small basis-set calculation, albeit minor ones. The B3LYP/6-31G(d) level of theory is hence deemed a reasonable compromise between cost and accuracy for these high-throughput calculations of frontier orbital properties, while the close correspondence between values for quantum mechanically optimized geometries and geometries extracted from MD simulations additionally serves as a validation of the IDTBR force fields.

The electronic couplings calculated for the as-cast and annealed thin-films of O-IDTBR and EH-IDTBR are presented in Fig. 10 as box plots for pairs with a distance of less than $4.5 \AA$ (dependence on distance and intermolecular orientation can be found in the SI, Figs. S12 and S13) [19]. These are compared to couplings calculated from MD runs at $300 \mathrm{~K}$ of their experimentally obtained crystal structures [22]. For both as-cast and annealed versions of O-IDBTR and EHIDTBR thin-films, the median values for electron coupling, i.e., electron transfer integrals, are centered around $11 \mathrm{meV}$ with slightly higher mean values of around $15 \mathrm{meV}$. No significant differences are observed between the two materials in thin-films, but for the crystal structures, EH-IDTBR exhibits only half the electron coupling strength of O-IDTBR. This correlates nicely with the results presented in Ref. [22] for geometries of single crystals deduced from x-ray diffraction at $173 \mathrm{~K}$; here, the four unique types of interactions considered in the O-IDTBR unit cell were calculated to have couplings of $9,51,58$, and $59 \mathrm{meV}$, whereas the two types in the EH-IDTBR unit cell were calculated to be 17 and $39 \mathrm{meV}$. These results were obtained using the same level of theory as was used herein, hence making them directly comparablehere, however, we include the effects of dynamics at $300 \mathrm{~K}$ and sample geometries from simulations spanning a large number of unit cells, which explains the wide distribution and the lower means of calculated values seen. Looking at the hole couplings, the tendencies are similar to the ones for the electron couplings, but the absolute values are in general lower by around $5 \mathrm{meV}$. Interestingly, the high structural order in O-IDTBR crystals yields hole couplings that are of the same strength as the crystal electron couplings, and almost $20 \mathrm{meV}$ higher than the O-IDTBR thin-film hole couplings,

TABLE III. Percentages of different interaction types relative to the total number of pairs with $\pi-\pi$ interactions closer than $4.5 \AA$.

\begin{tabular}{|c|c|c|c|c|c|c|}
\hline \multirow{2}{*}{$\begin{array}{l}\text { Amount }(\%) \\
\text { type }\end{array}$} & \multicolumn{3}{|c|}{ O-IDTBR } & \multicolumn{3}{|c|}{ EH-IDTBR } \\
\hline & as-cast & anneal. & crystal & as-cast & anneal. & crystal \\
\hline RH-RH & 8.6 & 4.3 & & 12.0 & 8.8 & \\
\hline BT-BT & 27.5 & 32.4 & 35.1 & 35.1 & 35.0 & \\
\hline IDT-IDT & 0.9 & 3.2 & & & & \\
\hline RH-BT & 33.3 & 25.9 & 61.4 & 42.9 & 41.9 & 100.0 \\
\hline RH-IDT & 13.5 & 20.0 & 3.5 & 4.7 & 6.2 & \\
\hline BT-IDT & 16.2 & 14.1 & & 5.2 & 8.1 & \\
\hline
\end{tabular}



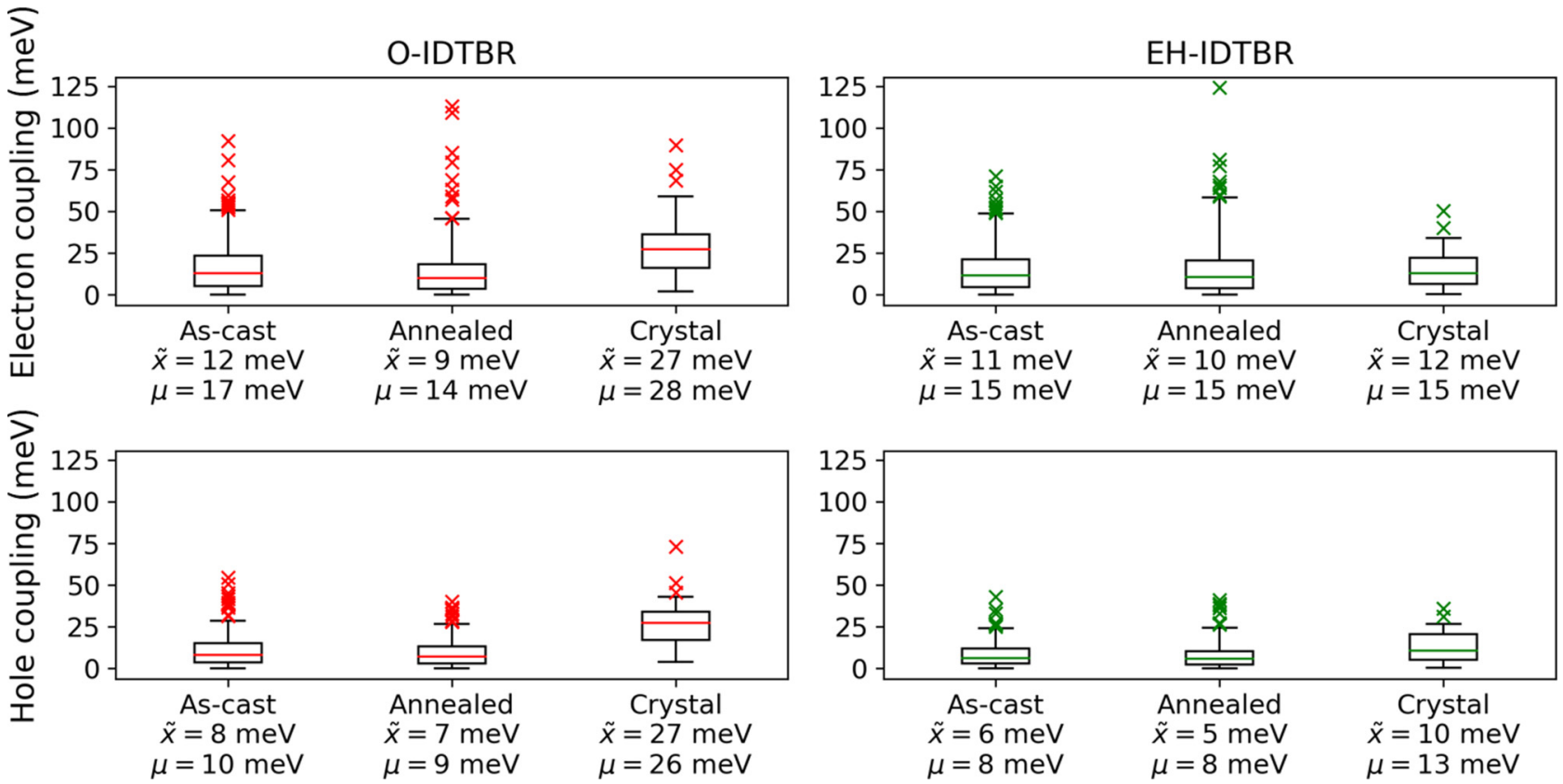

FIG. 10. Box plots of electronic couplings for electron (top row) and hole (bottom row) transfer (also referred to as effective electron/hole transfer integrals, $J_{e / h}$ ) for pairs stacked closer than $4.5 \AA$ in each of the simulated O-IDTBR (left column) and EH-IDTBR (right column) as-cast and annealed thin-films on $\mathrm{SiO}_{2}$ substrates compared to the corresponding couplings in their simulated crystal structures. Phase dependency is not considered (absolute values presented). The top 5\% of the values are plotted as outliers (red and green $\times$ 's). Median, $\tilde{x}$, and arithmetic mean, $\mu$, are indicated for each dataset.

whereas the hole couplings for EH-IDTBR crystals are only slightly larger than for the disordered thin-films.

To rationalize the above findings, the different types of close interactions (i.e., $\pi$-stacks, cf. Sec. III A) were analyzed and listed in Table III as percentages relative to the total number of pairs extracted, and in Tables IV and V as the mean electron and hole coupling strengths in meV, respectively. First and foremost, it is a clear tendency in Table III that close interactions of types BT-BT and RH-BT are the most frequent, both in the thin-films and in the crystals. This is expected to be beneficial to the electron transfer properties due to the molecular design of IDTBR with an electron donating central IDT unit flanked by electron withdrawing BT and RH units. As seen in the right panel of Fig. 9, the LUMO of IDTBR is localized mainly on the BT unit, and the electron coupling data in Table IV are indeed consistent with this, showing stronger couplings for interaction types involving the BT unit with a tendency of BT-BT interactions being the strongest in

TABLE IV. Mean electron coupling, $\mu\left(J_{e}\right)$, in meV of different interaction types of pairs with $\pi-\pi$ interactions closer than $4.5 \AA$.

\begin{tabular}{|c|c|c|c|c|c|c|}
\hline \multirow{2}{*}{$\begin{array}{l}\mu\left(J_{e}\right)(\mathrm{meV}) \\
\text { type }\end{array}$} & \multicolumn{3}{|c|}{ O-IDTBR } & \multicolumn{3}{|c|}{$\underline{\text { EH-IDTBR }}$} \\
\hline & as-cast & anneal. & crystal & as-cast & anneal. & crystal \\
\hline RH-RH & 16 & 6 & & 9 & 13 & \\
\hline BT-BT & 23 & 20 & 25 & 18 & 20 & \\
\hline IDT-IDT & 15 & 8 & & & & \\
\hline RH-BT & 16 & 17 & 31 & 15 & 14 & 15 \\
\hline RH-IDT & 14 & 8 & & 23 & 4 & \\
\hline BT-IDT & 13 & 12 & & 15 & 16 & \\
\hline
\end{tabular}

the thin-films. However, in the O-IDTBR crystal, the RH-BT interaction is significantly stronger than the same type in the EH-IDTBR crystal even though the individual geometries are very similar (parallel backbones, parallel $\pi$-planes; representative structures visualized in Fig. 11) - the only apparent difference is the relative orientation of the BT unit with respect to the RH and IDT units and the resulting intermolecular stacking. When the RH oxo group of a molecule A points in the direction of the BT sulfur of a molecule B (as is the case for EH-IDTBR crystals), the LUMO-LUMO overlap in the $\pi$-stack of molecule A and B is not as favorable as when the RH thioketone points in the direction of the BT sulfur (as is the case for O-IDTBR crystals), which leads to, on average, electronic couplings of only half the strength for both electrons (see Table IV) and holes (see Table V). In Ref. [22], the electron couplings for exactly these types of close interactions were found to be $9 \mathrm{meV}$ for O-IDTBR and $39 \mathrm{meV}$ for EH-IDTBR based on the experimentally

TABLE V. Mean hole coupling, $\mu\left(J_{h}\right)$, in meV of different interaction types of pairs with $\pi-\pi$ interactions closer than $4.5 \AA$.

\begin{tabular}{|c|c|c|c|c|c|c|}
\hline \multirow{2}{*}{$\begin{array}{l}\mu\left(J_{h}\right)(\mathrm{meV}) \\
\text { type }\end{array}$} & \multicolumn{3}{|c|}{ O-IDTBR } & \multicolumn{3}{|c|}{$\underline{\text { EH-IDTBR }}$} \\
\hline & as-cast & anneal. & crystal & as-cast & anneal. & crystal \\
\hline RH-RH & 8 & 4 & & 7 & 10 & \\
\hline BT-BT & 13 & 12 & 16 & 8 & 7 & \\
\hline IDT-IDT & 26 & 1 & & & & \\
\hline RH-BT & 9 & 9 & 32 & 9 & 9 & 13 \\
\hline RH-IDT & 14 & 9 & & 8 & 7 & \\
\hline BT-IDT & 9 & 7 & & 11 & 7 & \\
\hline
\end{tabular}



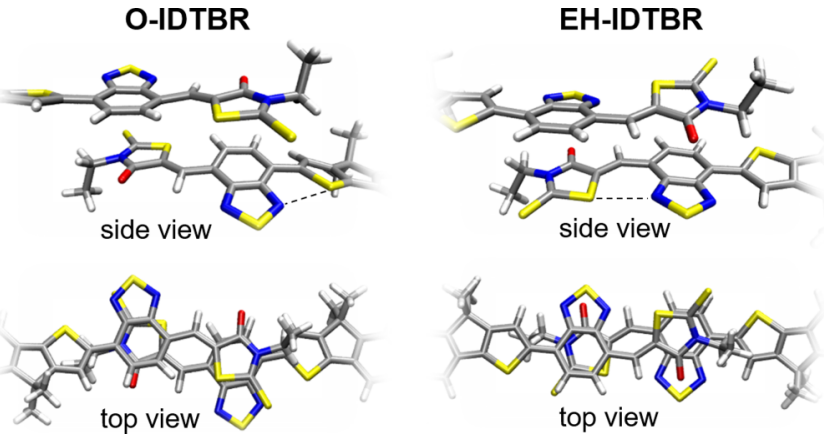

FIG. 11. Representative geometries of the RH-BT type of close interactions in O-IDTBR and EH-IDTBR crystal structures. The relative alignment of the $\mathrm{BT}$ unit to the $\mathrm{RH}$ and IDT units strongly influences the strength of the electronic coupling between the molecules.

determined crystal structures at $173 \mathrm{~K}$. We were able to reproduce these results, and it thus remains that either the consideration of dynamics and sampling at $300 \mathrm{~K}$ and/or the force fields themselves are the causes of this qualitative change in relative coupling strength. The latter was addressed in Sec. II D, which showed a good correspondence between the experimental crystal structures and the simulated crystal structures, and the inclusion of dynamics and the sampling from larger simulations thus seem to be key to predicting the charge-transport properties of molecular ensembles, even when crystal structures are available, as the electronic transfer integrals are sensitive to the structural changes induced by temperature.

Looking at the BT-RH stacking in the thin-films, our analysis (cf. the SI, Fig. S10) [19] shows that little to no difference between O-IDTBR and EH-IDTBR can be observed, both for as-cast and annealed crystals, with both materials predominantly exhibiting the BT-RH stacking geometry seen for $\mathbf{O}$ IDTBR crystals (left column of Fig. 11). This is expected due to this stacking geometry involving two IDTBR molecules that are both in their minimum energy configurations with respect to the torsional angle between BT and RH (cf. Figs. 3 and 11). The structural ordering seen in the EH-IDTBR crystal is thus not present to any significant degree in the thin-film simulations. It is important to note that although our findings for the crystal simulations are consistent with the experimental findings of higher mobilities in O-IDTBR OTFTs than in EH-IDTBR OTFTs [22], the simulation times reachable with atomistic MD are not sufficient to induce any significant crystallinity in the IDTBR thin-film simulations. It is apparent from the coupling calculations that an increased crystallinity would indeed be beneficial for the charge-transport properties of O-IDTBR thin-films when comparing the thin-film simulations to the crystal simulations. O-IDTBR has by differential scanning calorimetry been shown to have an exothermic crystallization transition with $T_{c}=115^{\circ} \mathrm{C}$, whereas EH-IDTBR has no such transition [20]. It can thus be concluded that the increased structural order upon annealing of O-IDTBR thin-films to more than $115^{\circ} \mathrm{C}$ is responsible for better chargetransport properties, but that this does not hold true for EHIDTBR thin-films, whose structural order and, in turn, chargetransport properties will seemingly not benefit significantly from annealing as seen in the SI, Fig. S4 [19], which is fully consistent with the simulation results presented herein.

\section{CONCLUSIONS}

In summary, in the present work we have devised a molecular dynamics solvent evaporation procedure that includes substrate and air-interface effects to model solution deposition of organic thin-films. The procedure is general and can be used in combination with any given substrate and for both atomistic and coarse-grained MD simulations. Here, we used our newly developed all-atom force fields for O-IDTBR and EH-IDTBR to investigate the structure-property relationships in their thin-films. It was found that the short-range structural properties, i.e., $\pi$-stacking, and the qualitative alignment effects relative to the substrate were accurately modeled, but it was evident that the time-scales feasible for atomistic MD, here on the order of $1 \mu \mathrm{s}$, were not sufficient to induce significant longer-range order in smallmolecule thin-films. Upon linking the structural findings to electronic properties, it was found that the $\pi$-stacking interactions of benzothiadiazole units were beneficial for the charge-transport properties of IDTBR thin-films, exhibiting high electronic transfer integrals even in disordered systems. This result is believed to be relevant for a range of $n$-type materials with similar molecular designs. Additionally, we saw indications of the terminal ethyl group on the rhodanine units inducing steric effects that are detrimental to the RH-BT type of stacking when the backbones are parallel, and future efforts in material development should focus on substituting this ethyl group with a methyl group or substituting the rhodanine unit altogether with planar, even stronger electron withdrawing units, which could additionally be beneficial for the long-wavelength absorption properties. We have become aware that this has recently been done in Ref. [63], where the rhodanine was substituted for a dicyano moiety, leading to small efficiency increases in organic solar cells.

Further work will focus on thin-film morphologies of organic solar cell active layer blends based on these IDTBR acceptors and relevant polymer donors. Additionally, a coarsegrained force field for IDTBR is currently being developed to investigate whether higher structural order can be obtained with bigger system sizes and longer simulation times.

\section{ACKNOWLEDGMENTS}

The authors acknowledge financial support from the H2020 European Research Council through the SEEWHI Consolidator grant, ERC-2015-CoG-681881. A.S.G. thanks the team responsible for the Niflheim supercomputer at the Technical University of Denmark, Department of Physics where all calculations were performed. A.S.G. thanks Jenny Nelson and Jarvist M. Frost for guidance and discussions regarding the electronic coupling calculations, Elisabeth Rice and Francesco Salerno for providing a script for the projective method, Anne A. Y. Guilbert and Drew Pearce for support on MD simulations during a half year secondment at Imperial College London, and Natalie Stingelin for facilitating it. A.S.G. acknowledges generous funding from Otto Mønsteds Fond (Grant No. 17-70-1617) in connection with this secondment. 
[1] N. Espinosa, M. Hosel, D. Angmo, and F. C. Krebs, Solar cells with one-day energy payback for the factories of the future, Energy Environ. Sci. 5, 5117 (2012).

[2] C. J. Emmott, A. Urbina, and J. Nelson, Environmental and economic assessment of ITO-free electrodes for organic solar cells, Sol. Energy Mater. Sol. Cells 97, 14 (2012).

[3] A. S. Gertsen, M. F. Castro, R. R. Søndergaard, and J. W. Andreasen, Scalable fabrication of organic solar cells based on non-fullerene acceptors, Flex. Print. Electron. 5, 014004 (2020).

[4] C. Yan, S. Barlow, Z. Wang, H. Yan, A. K. Jen, S. R. Marder, and $X$. Zhan, Non-fullerene acceptors for organic solar cells, Nat. Rev. Mater. 3, 18003 (2018).

[5] G. Zhang, J. Zhao, P. C. Y. Chow, K. Jiang, J. Zhang, Z. Zhu, J. Zhang, F. Huang, and H. Yan, Nonfullerene acceptor molecules for bulk heterojunction organic solar cells, Chem. Rev. 118, 3447 (2018).

[6] Q. Liu, Y. Jiang, K. Jin, J. Qin, J. Xu, W. Li, J. Xiong, J. Liu, Z. Xiao, K. Sun, S. Yang, X. Zhang, and L. Ding, 18\% efficiency organic solar cells, Sci. Bull. 65, 272 (2020).

[7] J. Hou, O. Inganäs, R. H. Friend, and F. Gao, Organic solar cells based on non-fullerene acceptors, Nat. Mater. 17, 119 (2018).

[8] N. Gasparini, M. Salvador, S. Strohm, T. Heumueller, I. Levchuk, A. Wadsworth, J. H. Bannock, J. C. de Mello, H.-J. Egelhaaf, D. Baran, I. McCulloch, and C. J. Brabec, Burn-in free nonfullerene-based organic solar cells, Adv. Energy Mater. 7, 1700770 (2017).

[9] H. Cha, J. Wu, A. Wadsworth, J. Nagitta, S. Limbu, S. Pont, Z. Li, J. Searle, M. F. Wyatt, D. Baran, J.-S. Kim, I. McCulloch, and J. R. Durrant, An efficient, "burn in" free organic solar cell employing a nonfullerene electron acceptor, Adv. Mater. 29, 1701156 (2017).

[10] D. Baran, R. S. Ashraf, D. A. Hanifi, M. Abdelsamie, N. Gasparini, J. A. Röhr, S. Holliday, A. Wadsworth, S. Lockett, M. Neophytou, C. J. M. Emmott, J. Nelson, C. J. Brabec, A. Amassian, A. Salleo, T. Kirchartz, J. R. Durrant, and I. McCulloch, Reducing the efficiency-stability-cost gap of organic photovoltaics with highly efficient and stable small molecule acceptor ternary solar cells, Nat. Mater. 16, 363 (2017).

[11] W. Zhao, D. Qian, S. Zhang, S. Li, O. Inganäs, F. Gao, and J. Hou, Fullerene-free polymer solar cells with over $11 \%$ efficiency and excellent thermal stability, Adv. Mater. 28, 4734 (2016).

[12] X. Du, T. Heumueller, W. Gruber, A. Classen, T. Unruh, N. Li, and C. J. Brabec, Efficient polymer solar cells based on nonfullerene acceptors with potential device lifetime approaching 10 years, Joule 3, 215 (2019).

[13] M. Jørgensen, K. Norrman, S. A. Gevorgyan, T. Tromholt, B. Andreasen, and F. C. Krebs, Stability of polymer solar cells, Adv. Mater. 24, 580 (2012).

[14] P. Cheng, H. Bai, N. K. Zawacka, T. R. Andersen, W. Liu, E. Bundgaard, M. Jørgensen, H. Chen, F. C. Krebs, and X. Zhan, Roll-coated fabrication of fullerene-free organic solar cells with improved stability, Adv. Sci. 2, 1500096 (2015).

[15] D. Baran, T. Kirchartz, S. Wheeler, S. Dimitrov, M. Abdelsamie, J. Gorman, R. S. Ashraf, S. Holliday, A. Wadsworth, N. Gasparini, P. Kaienburg, H. Yan, A. Amassian, C. J. Brabec, J. R. Durrant, and I. McCulloch, Reduced voltage losses yield $10 \%$ efficient fullerene free organic solar cells with $>1 \mathrm{~V}$ open circuit voltages, Energy Environ. Sci. 9, 3783 (2016).

[16] N. Li, J. D. Perea, T. Kassar, M. Richter, T. Heumueller, G. J. Matt, Y. Hou, N. S. Güldal, H. Chen, S. Chen, S. Langner, M. Berlinghof, T. Unruh, and C. J. Brabec, Abnormal strong burnin degradation of highly efficient polymer solar cells caused by spinodal donor-acceptor demixing, Nat. Commun. 8, 14541 (2017).

[17] F. D. Angelis, R. Berardi, J. Bisquert, J.-L. Bredas, G. D’Avino, N. Greenham, C. Groves, E. Hontz, T. Kirchartz, R. Marcus, D. McMahon, L. Muccioli, J. Nelson, S. Orlandi, M. Pastore, A. Pizzirusso, M. Ricci, C. Risko, O. Roscioni, T. V. Voorhis, A. Walker, S. Yost, and C. Zannoni, Multiscale modeling of organic and hybrid photovoltaics, in Topics in Current Chemistry, edited by D. Beljonne and J. Cornil (Springer-Verlag, Berlin, 2014), Vol. 352, pp. 1-400.

[18] Q.-Q. Pan, S.-B. Li, Y.-C. Duan, Y. Wu, J. Zhang, Y. Geng, L. Zhao, and Z.-M. Su, Exploring what prompts ITIC to become a superior acceptor in organic solar cell by combining molecular dynamics simulation with quantum chemistry calculation, Phys. Chem. Chem. Phys. 19, 31227 (2017).

[19] See Supplemental Material at http://link.aps.org/supplemental/ 10.1103/PhysRevMaterials.4.075405 for GROMACS force field files for O-IDTBR and EH-IDTBR for use with OPLS-AA, complete compound names, detailed force-field parametrization procedures and tables of parameters, experimental details and GIWAXS data for $130^{\circ} \mathrm{C}$ annealed thin-films, details of the structural analysis, and dependence of electronic coupling on intermolecular distance and relative orientation.

[20] S. Holliday, R. S. Ashraf, A. Wadsworth, D. Baran, S. A Yousaf, C. B. Nielsen, C.-H. Tan, S. D. Dimitrov, Z. Shang, N. Gasparini, M. Alamoudi, F. Laquai, C. J. Brabec, A. Salleo, J. R. Durrant, and I. McCulloch, High-efficiency and air-stable P3HT-based polymer solar cells with a new non-fullerene acceptor, Nat. Commun. 7, 11585 (2016).

[21] A. Wadsworth, R. S. Ashraf, M. Abdelsamie, S. Pont, M. Little, M. Moser, Z. Hamid, M. Neophytou, W. Zhang, A. Amassian, J. R. Durrant, D. Baran, and I. McCulloch, Highly efficient and reproducible nonfullerene solar cells from hydrocarbon solvents, ACS Energy Lett. 2, 1494 (2017).

[22] H. Bristow, K. J. Thorley, A. J. P. White, A. Wadsworth, M. Babics, Z. Hamid, W. Zhang, A. F. Paterson, J. Kosco, J. Panidi, T. D. Anthopoulos, and I. McCulloch, Impact of nonfullerene acceptor side chain variation on transistor mobility, Adv. Electron. Mater. 5, 1900344 (2019).

[23] M. Yoneya, H. Minemawari, T. Yamada, and T. Hasegawa, Interface-mediated self-assembly in inkjet printing of singlecrystal organic semiconductor films, J. Phys. Chem. C 121, 8796 (2017).

[24] T. E. Gartner and A. Jayaraman, Modeling and simulations of polymers: A roadmap, Macromol. 52, 755 (2019).

[25] E. Jankowski, H. S. Marsh, and A. Jayaraman, Computationally linking molecular features of conjugated polymers and fullerene derivatives to bulk heterojunction morphology, Macromolecules 46, 5775 (2013).

[26] M. M. Henry, M. L. Jones, S. D. Oosterhout, W. A. Braunecker, T. W. Kemper, R. E. Larsen, N. Kopidakis, M. F. Toney, D. C. Olson, and E. Jankowski, Simplified models for accelerated 
structural prediction of conjugated semiconducting polymers, J. Phys. Chem. C 121, 26528 (2017).

[27] Y.-B. Lan, P.-H. Sher, C.-K. Lee, C.-W. Pao, C.-S. Tsao, Y.C. Huang, P.-T. Huang, C.-I. Wu, and J.-K. Wang, Revealing ordered polymer packing during freeze-drying fabrication of a bulk heterojunction poly(3-hexylthiophene-2, 5-diyl):[6, 6]phenyl-c61-butyric acid methyl ester layer: In situ optical spectroscopy, molecular dynamics simulation, and X-ray diffraction, J. Phys. Chem. C 121, 14826 (2017).

[28] G. Han, Y. Yi, and Z. Shuai, From molecular packing structures to electronic processes: Theoretical simulations for organic solar cells, Adv. Energy Mater. 8, 1702743 (2018).

[29] S. Peter, H. Meyer, and J. Baschnagel, Molecular dynamics simulations of concentrated polymer solutions in thin film geometry. II. Solvent evaporation near the glass transition, J. Chem. Phys. 131, 014903 (2009).

[30] V. Negi, A. Lyulin, and P. Bobbert, Solvent-dependent structure formation in drying P3HT:PCBM films studied by molecular dynamics simulations, Macromol. Theor. Simul. 25, 550 (2016).

[31] C.-K. Lee and C.-W. Pao, Nanomorphology evolution of $\mathrm{P} 3 \mathrm{HT} / \mathrm{PCBM}$ blends during solution-processing from coarsegrained molecular simulations, J. Phys. Chem. C 118, 11224 (2014).

[32] C.-K. Lee and C.-W. Pao, Multiscale molecular simulation of solution processing of SMDPPEH:PCBM small-molecule organic solar cells, ACS Appl. Mater. Interfaces 8, 20691 (2016).

[33] S. J. Marrink, H. J. Risselada, S. Yefimov, D. P. Tieleman, and A. H. de Vries, The MARTINI force field: Coarse grained model for biomolecular simulations, J. Phys. Chem. B 111, 7812 (2007).

[34] R. Alessandri, J. J. Uusitalo, A. H. de Vries, R. W. A. Havenith, and S. J. Marrink, Bulk heterojunction morphologies with atomistic resolution from coarse-grain solvent evaporation simulations, J. Am. Chem. Soc. 139, 3697 (2017).

[35] G. Han, X. Shen, R. Duan, H. Geng, and Y. Yi, Revealing the influence of the solvent evaporation rate and thermal annealing on the molecular packing and charge transport of DPP(TBFu 2 , J. Mater. Chem. C 4, 4654 (2016).

[36] G. Han, Y. Guo, X. Ma, and Y. Yi, Atomistic insight into donor/acceptor interfaces in high-efficiency nonfullerene organic solar cells, Solar RRL 2, 1800190 (2018).

[37] A. F. Marmolejo-Valencia, Z. Mata-Pinzón, L. Dominguez, and C. Amador-Bedolla, Atomistic simulations of bulk heterojunctions to evaluate the structural and packing properties of new predicted donors in OPVs, Phys. Chem. Chem. Phys. 21, 20315 (2019).

[38] M. J. Abraham, T. Murtola, R. Schulz, S. Páll, J. C. Smith, B. Hess, and E. Lindahl, GROMACS: High performance molecular simulations through multi-level parallelism from laptops to supercomputers, Software X 1-2, 19 (2015).

[39] W. L. Jorgensen, D. S. Maxwell, and J. Tirado-Rives, Development and testing of the OPLS all-atom force field on conformational energetics and properties of organic liquids, J. Am. Chem. Soc. 118, 11225 (1996).

[40] G. A. Kaminski, R. A. Friesner, J. Tirado-Rives, and W. L. Jorgensen, Evaluation and reparametrization of the OPLS-AA force field for proteins via comparison with accurate quantum chemical calculations on peptides, J. Phys. Chem. B 105, 6474 (2001).
[41] R. T. Cygan, J.-J. Liang, and A. G. Kalinichev, Molecular models of hydroxide, oxyhydroxide, and clay phases and the development of a general force field, J. Phys. Chem. B 108, 1255 (2004).

[42] S. Leroch and M. Wendland, Simulation of forces between humid amorphous silica surfaces: A comparison of empirical atomistic force fields, J. Phys. Chem. C 116, 26247 (2012)

[43] O. M. Roscioni, G. D’Avino, L. Muccioli, and C. Zannoni, Pentacene crystal growth on silica and layer-dependent stepedge barrier from atomistic simulations, J. Phys. Chem. Lett. 9, 6900 (2018).

[44] C. Caleman, P. J. van Maaren, M. Hong, J. S. Hub, L. T. Costa, and D. van der Spoel, Force field benchmark of organic liquids: Density, enthalpy of vaporization, heat capacities, surface tension, isothermal compressibility, volumetric expansion coefficient, and dielectric constant, J. Chem. Theor. Comput. 8, 61 (2012).

[45] S. Strohm, F. Machui, S. Langner, P. Kubis, N. Gasparini, M. Salvador, I. McCulloch, H.-J. Egelhaafa, and C. J. Brabec, P3HT: Non-fullerene acceptor based large area, semitransparent PV modules with power conversion efficiencies of $5 \%$, processed by industrially scalable methods, Energy Environ. Sci. 11, 2225 (2018).

[46] J. H. Yang, T. Ryua, Y. Lansac, Y. H. Jang, and B. H. Lee, Shear stress-induced enhancement of the piezoelectric properties of PVDF-TrFE thin films, Org. Electron. 28, 67 (2016).

[47] M. J. Frisch, G. W. Trucks, H. B. Schlegel, G. E. Scuseria, M. A. Robb, J. R. Cheeseman, G. Scalmani, V. Barone, G. A. Petersson, H. Nakatsuji et al., GAUSSIAN 16 Revision A.03, 2016. GAUSSIAN Inc., Wallingford CT.

[48] C. Lee, W. Yang, and R. G. Parr, Development of the ColleSalvetti correlation-energy formula into a functional of the electron density, Phys. Rev. B 37, 785 (1988).

[49] A. D. Becke, Density functional thermochemistry. III. The role of exact exchange, J. Chem. Phys. 98, 5648 (1993).

[50] P. J. Stephens, F. J. Devlin, C. F. Chabalowski, and M. J. Frisch, $\mathrm{Ab}$ initio calculation of vibrational absorption and circular dichroism spectra using density functional force fields, J. Phys. Chem. 98, 11623 (1994).

[51] J.-D. Chai and M. Head-Gordon, Systematic optimization of long-range corrected hybrid density functionals, J. Chem. Phys. 128, 084106 (2008).

[52] J.-D. Chai and M. Head-Gordon, Long-range corrected hybrid density functionals with damped atom-atom dispersion corrections, Phys. Chem. Chem. Phys. 10, 6615 (2008).

[53] R. Krishnan, J. S. Binkley, R. Seeger, and J. A. Pople, Selfconsistent molecular orbital methods. XX. A basis set for correlated wave functions, J. Chem. Phys. 72, 650 (1980).

[54] J. S. Binkley, J. A. Pople, and W. J. Hehre, Self-consistent molecular orbital methods. 21. Small split-valence basis sets for first-row elements, J. Am. Chem. Soc. 102, 939 (1980).

[55] A. A. Y. Guilbert, J. M. Frost, T. Agostinelli, E. Pires, S. Lilliu, J. E. Macdonald, and J. Nelson, Influence of bridging atom and side chains on the structure and crystallinity of cyclopentadithiophene-benzothiadiazole polymers, Chem. Mater. 26, 1226 (2014).

[56] M. J. Robertson, J. Tirado-Rives, and W. L. Jorgensen, Improved peptide and protein torsional energetics with the OPLSAA force field, J. Chem. Theor. Comput. 11, 3499 (2015). 
[57] M. J. Abraham, D. van der Spoel, E. Lindahl, B. Hess, and the GROMACS development team, GROMACS User Manual version 2016.3, 2017.

[58] C. F. Macrae, I. J. Bruno, J. A. Chisholm, P. R. Edgington, P. McCabe, E. Pidcock, L. Rodriguez-Monge, R. Taylor, J. van de Streek, and P. A. Wood, Mercury CSD 2.0 - new features for the visualization and investigation of crystal structures, J. Appl. Crystallogr. 41, 466 (2008).

[59] B. Kang, M. Jang, Y. Chung, H. Kim, S. K. Kwak, J. H. Oh, and K. Cho, Enhancing 2D growth of organic semiconductor thin films with macroporous structures via a small-molecule heterointerface, Nat. Commun. 5, 4752 (2014).

[60] Z. Ma, H. Geng, D. Wang, and Z. Shuai, Influence of alkyl sidechain length on the carrier mobility in organic semiconductors:
Herringbone vs pi-pi stacking, J. Mater. Chem. C 4, 4546 (2016).

[61] J. Kirkpatrick, Calculating intermolecular charge transport parameters in conjugated materials, $\mathrm{Ph} . \mathrm{D}$. thesis, Imperial College London, 2007.

[62] E. Rice, Computational modelling of electronic states, charge transfer and charge transport in organic semiconductors. Ph.D. thesis, Imperial College London, 2018.

[63] A. Wadsworth, H. Bristow, Z. Hamid, M. Babics, N. Gasparini, C. W. Boyle, W. Zhang, Y. Dong, K. J. Thorley, M. Neophytou, R. S. Ashraf, J. R. Durrant, D. Baran, and I. McCulloch, End group tuning in acceptor-donor-acceptor nonfullerene small molecules for high fill factor organic solar cells, Adv. Funct. Mater. 29, 1808429 (2019). 